$15.2,09.5$

\title{
Применение метода прямого оптического гетеродинирования для измерения твердости методом Либа
}

\author{
(С) Т.В. Казиева, К.Л. Губский, А.П. Кузнецов, Р.Д. Глухов \\ Национальный исследовательский ядерный университет „МИФИ“, Москва, Россия \\ E-mail: glizerogen@gmail.com
}

Поступило в Редакцию 10 июня 2019 г.

В окончательной редакции 10 июня 2019 г.

Принято к публикации 17 июня 2019 г.

\begin{abstract}
Описана портативная система для измерения скорости индентора в твердомерах, реализованных по методу Либа. Разработанная система представляет собой волоконный интерферометр, источником излучения в котором является полупроводниковый лазер с длиной волны $1550 \mathrm{~nm}$. Представленные результаты экспериментов подтверждают способность системы измерять скорости индентора с неопределенностью $0.001 \mathrm{~m} / \mathrm{s}$, что удовлетворяет требованиям стандартов к данной методике измерения твердости.
\end{abstract}

Ключевые слова: волоконный интерферометр, PDV, измерение твердости, метод Либа.

DOI: 10.21883/PJTF.2019.19.48309.17918

Существует множество способов определения твердости, в зависимости от временно́го характера приложения нагрузки и измерения сопротивления вдавливанию индентора выделяют статические (плавное нагружение образца) и динамические (действие на образец с определенной кинетической энергией) методы. В отличие от статических методов динамические позволяют получить дополнительную информацию об ответном поведении материалов, например об эластических свойствах. Один из наиболее распространенных сейчас динамических методов - это метод Либа [1].

Метод определения твердости по Либу основан на изменении отношения скоростей ударника до удара о поверхность образца из исследуемого материала и после отскока. Скорости падения-отскока в настоящее время определяются посредством измерения ЭДС, наводимой в катушке индуктивности постоянным магнитом, закрепленным на ударнике вместе с твердосплавным шариком, который выступает в роли индентора. Метрологическое обеспечение метода Либа в США и Европе регламентируется стандартами DIN 50156, ASTM A956, ISO/DIS 16859. До настоящего времени в Российской Федерации отсутствует стандарт на метод измерения твердости по Либу, но утвержден план введения [2]. Для проведения калибровок систем, работающих по данной методике, используются лазерные интерферометры. С их помощью измеряется скорость индентора в момент удара об исследуемую поверхность, при этом точность должна составлять $\sim 10^{-3} \mathrm{~m} / \mathrm{s}$. Однако существующие на данный момент установки для калибровки представляют собой крупногабаритные лабораторные комплексы, работающие на открытом луче и сложные в настройке [3].

Авторами предложен метод калибровки твердомеров с помощью компактного волоконного лазерного интерферометра, который позволяет проводить измерения скорости ударника на всем пути его движения с высокой точностью.

Разработанная система реализована по методике PDV (Photonic Doppler Velocimetry), основанной на прямом оптическом гетеродинировании с прямым преобразованием доплеровских сигналов. Преимуществами данной методики являются помехозащищенность, высокая точность и простота в реализации [4].

Схема изготовленного интерферометра приведена на рис. 1. В качестве источника излучения используется полупроводниковый лазер 1 с длиной волны $1550 \mathrm{~nm}$. Проходя первый волоконный светоделитель 2, излучение лазера разделяется на два плеча: опорное и измерительное, соотношение мощностей в плечах 1/9. Далее в измерительном плече установлен циркулятор 3, к выходному каналу которого присоединена градиентная линза 4 , благодаря ей излучение подводится к индентору 5 и фокусируется на движущейся поверхности.

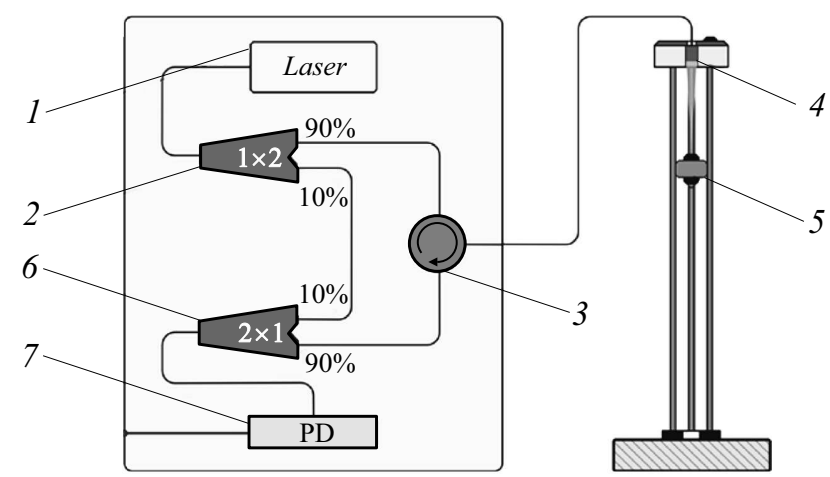

Рис. 1. Схема интерферометра для измерения скорости ударника. 1 - источник лазерного излучения, $2-$ светоделитель с коэффициентом $1 / 9,3$ - циркулятор, 4 - градиентная линза, 5 - индентор, 6 - оптоволоконный смеситель, 7 фотодетектор. 
Та же линза собирает часть излучения, отраженного движущимся объектом. Отраженное объектом излучение, проходя через циркулятор и оптоволоконный смеситель 6, объединяется с опорным сигналом и далее по волокну подводится к фотоприемнику 7, полученный сигнал регистрируется осциллографом.

Для восстановления динамики изменения частоты регистрируемого сигнала применяется оконное фурьепреобразование. Зарегистрированный сигнал разбивается на отрезки с характерным шагом (шириной окна). По спектральной плотности мощности, получаемой после фурье-преобразования сигнала в каждом окне, методом аппроксимации функцией Гаусса определяется максимальное значение частоты. Скорость объекта в каждом окне рассчитывается по формуле

$$
V(t)=f_{d} \lambda / 2
$$

где $\lambda$ - длина волны используемого лазера, $f_{d}-$ частота биений.

При скорости $3 \mathrm{~m} / \mathrm{s}$ частота биений $f_{d}=3.87 \mathrm{MHz}$. Используемая модель фотоприемника HFBR-2316 с полосой пропускания $100 \mathrm{MHz}$ не ограничивает регистрируемую частоту.

При выполнении оконного фурье-преобразования возникает неопределенность [5]: $\sigma_{f} \sigma_{t} \geq 1 / 4 \pi$, где $\sigma_{t}-$ неопределенность времени, $\sigma_{f}$ - неопределенность частоты сигнала. Поскольку в соответствии со стандартом неопределенность измерения скорости должна составлять величину $0.0025 \mathrm{~m} / \mathrm{s}$, неопределенность измерения частоты сигнала $-3.23 \mathrm{kHz}$, что ограничивает неопределенность по времени величиной $25 \mu \mathrm{s}$.

При разбиении сигнала на окна уменьшение длины окна приведет к росту погрешности при измерении частоты, а ее увеличение - к уменьшению временно́го разрешения.

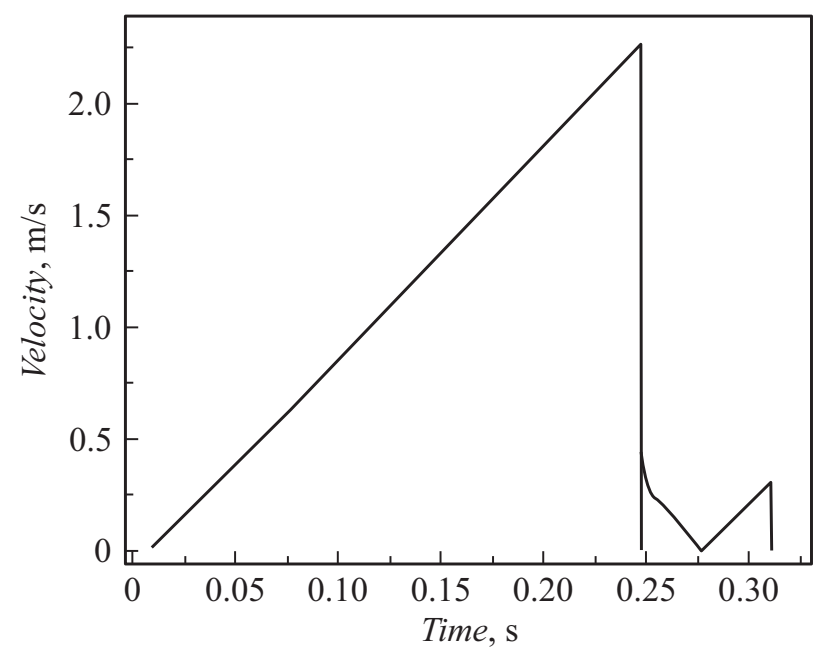

Рис. 2. Динамика скорости бойка.
Значение дисперии частотного представления оконной функции Хэмминга и расчеты погрешности скорости с учетом первого слагаемого в формуле (2)

\begin{tabular}{c|c|c}
\hline Размер окна $N$ (количество точек) & $\sigma_{f}, \mathrm{kHz}$ & $\pm \Delta v, \mathrm{~m} / \mathrm{s}$ \\
\hline 128 & 43 & 0.033 \\
512 & 10 & 0.008 \\
1024 & 5 & 0.0044 \\
2048 & 2.5 & 0.0027 \\
4096 & 1.3 & 0.0022
\end{tabular}

Абсолютная погрешность скорости рассчитывается по формуле

$$
\Delta v=\sqrt{\left(\frac{f_{d}}{2}\right)^{2} \sigma_{\lambda}^{2}+\left(\frac{\lambda}{2}\right)^{2} \sigma_{f}^{2}} .
$$

Первое слагаемое состоит из двух множителей: $f_{d}-$ максимальная частота интерференционного сигнала (для данной скорости эта величина не превосходит $4 \mathrm{MHz}$ ), $\sigma_{\lambda}$ - отклонение длины волны лазера, которое составляет $1 \mathrm{~nm}$. Таким образом,

$$
\frac{f_{d}}{2} \sigma_{\lambda}=2 \cdot 10^{-3}
$$

Лазер имеет длину волны $\lambda=1550 \mathrm{~nm}, \sigma_{f}-$ среднеквадратичное отклонение, вносимое математической обработкой экспериментальных данных, эта величина зависит от типа окна и количества точек в нем. Полный частотный интервал определения окна равен $\langle f\rangle \pm 2 \sigma_{f}$ (на данном частотном интервале энергия сигнала составляет не менее 95\% от его полной энергии).

Для минимальной частоты дискретизации ( 10 MS/s по теореме Котельникова) в таблице приведена неопределенность измерения скорости с учетом первого слагаемого в формуле (2), расчеты проведены для окна Хэмминга.

Исходя из данных таблицы можно заключить, что минимально необходимое количество точек для достижения требуемой неопределенности составляет 4096 при минимальной частоте дискретизации (т.е. временно́е разрешение составляет $0.2 \mathrm{~ms}$ ).

Первые работы по испытаниям разработанной системы для измерения скорости бойка твердомера были проведены на установке, показанной на рис. 1. Прототип твердомера представлял собой вертикальную конструкцию высотой около $0.3 \mathrm{~m}$, что обеспечивает разгон типового ударника до скорости $2.4 \mathrm{~m} / \mathrm{s}$. Известно, что по стандартам скорости, принятые для серийно выпускаемых образцов твердомеров, составляют 1.4, 2.05 и до $3 \mathrm{~m} / \mathrm{s}$; следовательно, данный образец позволяет провести первичный тест интерферометра.

Для регистрации скорости ударника градиентная линза в специальном держателе размещалась в верхней точке конструкции, выше, чем точка начала движения. Подводимое излучение отражалось от верхней плоской 
поверхности ударника. Регистрируемый сигнал записывался на осциллограф и после обрабатывался с помощью специального программного обеспечения.

На рис. 2 представлены результаты, полученные при одном из пусков системы. На данном рисунке можно выделить точки начала движения, область равномерного ускорения до $0.24 \mathrm{~s}$, а также момент отскока от поверхности исследуемого материала. Максимальная скорость перед падением на исследуемый материал составляет $2.259 \pm 0.002 \mathrm{~m} / \mathrm{s}$, скорость после отскока $0.461 \pm 0.002 \mathrm{~m} / \mathrm{s}$. Следовательно, данная методика позволяет осуществлять наблюдение на всем пути индентоpa, а получаемая при этом погрешность укладывается в заявленные требования стандартов.

Разработанная система позволяет измерять временну́ю зависимость скорости объекта от начала движения и до второго отскока от поверхности. Это дает возможность провести полноценный расчет твердости материала. Если же в конструкцию твердомера встроена система для регистрации скорости (например, индукционный датчик), то интерферометр можно использовать для калибровки.

\section{Финансирование работы}

Работа выполнена при поддержке Российского научного фонда (грант № 17-72-20164).

\section{Конфликт интересов}

Авторы заявляют, что у них нет конфликта интересов.

\section{Список литературы}

[1] Leeb D. // VDI-Report. 1978. N 308. P. 123-128.

[2] Syasko V.A., Gogolinskiy K.V., Nikazov A.A. // NDT World. 2017. V. 20. N 1. P. 16-19.

[3] Schwenk D. Leeb hardness-calibration-machine // IMEKO2010. TC3, TC5 and TC22 Conf. Metrology. Thailand, 2010. P. 369-372.

[4] Gorbashova M., Burdonskiy I., Gubskiy K., Kuznetsov A., Ramazanov A., Lukyanov K., Leonov A., Makarov K., Timofeyev I., Yufa V. // J. Phys.: Conf. Ser. 2017. V. 941. P. 012002.

[5] Cohen L. Time-frequency analysis. N.J.: Prentice Hall PTR, 1995. $300 \mathrm{p}$. 